\title{
Molecular gas around the Wolf-Rayet star WR 18 (WN4)
}

\author{
Anthony P. Marston \\ SIRTF Science Center, California Institute of Technology, \\ MS 220-6, 1200 East California Blvd., Pasadena, CA 91125, USA
}

\begin{abstract}
Optically observed ring nebulae and $\mathrm{H}$ I cavities around Wolf-Rayet stars have enabled us to obtain information on the history of mass-loss associated with these massive evolved stars. However, such studies have left a number of unanswered questions regarding the amount of mass-loss and the conditions of the stars during a sequence of mass-loss phases. Here we discuss the molecular gas environments of the WR star WR 18, which has an associated optical ring nebula NGC 3199. Our observations show that significant amounts of molecular gas appear close to and associated with the star. Mapping of molecular CO near the star shows that molecular materials appear to substantially avoid areas of optical emission and, instead, form a distorted clumpy shell interior to NGC 3199. Molecular emission lines are broader than lines seen in the interstellar medium and suggest the shell is composed of ejecta. This is further corroborated by the enhanced abundances of molecules containing $\mathrm{C}, \mathrm{N}$ and $\mathrm{O}$. Implications of the observations for the evolution of WR 18 are discussed.
\end{abstract}

\section{Introduction}

WR 18 (HD 89358) is a WN4 star that shows no signs of binarity. It is situated near the projected center of an optically bright ring nebula arc, NGC 3199. The main portion of the ring nebula is situated approximately $5^{\prime}$ to the west of the star. On a larger scale, NGC 3199. appears to be associated with a large $\left(>20^{\prime}\right.$ across) kidney-shaped shell, visible with IRAS-HIRES images. Both Hipparcos and Tycho data indicate that the star is moving to the north west at around $5 \mathrm{mas} \mathrm{yr}^{-1}$. For a distance of $2.2 \mathrm{kpc}$ (van der Hucht 2001) this equates to a tangential velocity of $60 \mathrm{~km} \mathrm{~s}^{-1}$.

As part of a continuing investigation of the gaseous environment of WR stars, we have mapped the surroundings of WR 18 in the ${ }^{12} \mathrm{CO} \mathrm{J}=1 \rightarrow 0$ emission line and searched for further molecular emission-line species in the direction of WR 18. Earlier observations of the region, including the area of NGC 3199 were reported by Marston (2001).

\section{Observations}

The molecular emission-line observations were made at the Swedish-ESO Submillimeter Telescope (SEST), La Silla, Chile, in April 2000. An undersampled map with pointings every $1^{\prime}$ in RA and Dec was obtained for the emission-lines of ${ }^{12} \mathrm{CO} \mathrm{J}=1 \rightarrow 0$ and ${ }^{12} \mathrm{CO} \mathrm{J}=2 \rightarrow 1$ (beam sizes of $45^{\prime \prime}$ and $23^{\prime \prime}$, respectively). The emisison-lines of $\mathrm{HCN}, \mathrm{HNC}, \mathrm{HCO}^{+}$and $\mathrm{CN}$ were also observed. A grid 


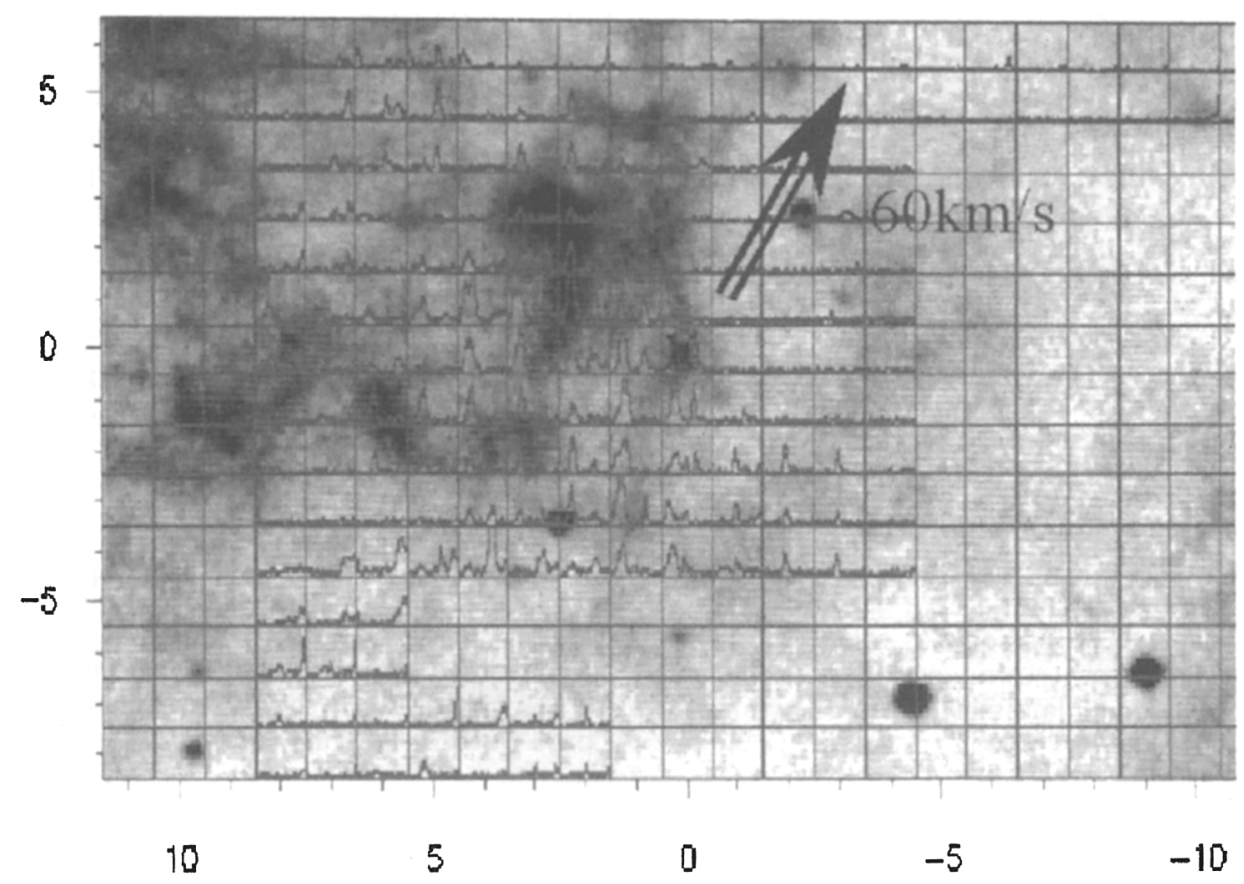

Figure 1. Grid of ${ }^{12} \mathrm{CO} \mathrm{J}=1 \rightarrow 0$ emission-line profiles overlaid on the $M S X$ $8.28 \mu \mathrm{m}$ image of the WR 18 region. The velocity range of the profiles is -12 to $+12 \mathrm{~km} \mathrm{~s}^{-1}$ (LSR). The proper motion of WR 18 is shown. Distance of the profiles from WR 18 is given in arcminutes along each edge of the figure.

of the profiles obtained is shown as an overlay on the MSX $8.28 \mu \mathrm{m}$ image in Figure 1. Profiles of the other observed emission-lines are presented by Marston (2001).

\section{Discussion}

WR 18 appears to lie on the edge of a slowly expanding, $\sim 5 \mathrm{~km} \mathrm{~s}^{-1}$, cocoon of clumpy molecular material that is approximately $5^{\prime}$ across. The existence of HCN emission suggests high densities $\left(>10^{4} \mathrm{~cm}^{-3}\right)$. Abundances of HCN and $\mathrm{CN}$ are substantially higher than the interstellar medium and suggest processed material, probably from the surface of the star, is in the molecular gas. The most likely origin of this material is a slow, cool wind phase in an earlier evolutionary phase of the star such as a red supergiant.

Acknowledgments. The research described in this paper was carried out at the Jet Propulsion Laboratory, California Institute of Technology, under a contract with the National Aeronautics and Space Administration.

\section{References}

Marston, A.P. 2001, ApJ 563, 875

van der Hucht, K.A. 2001, New Astron. Reviews 45, 135 